\title{
Validación y fiabilidad del instrumento «escala de percepción de competencias docente en educación física en la atención de las personas en situación de discapacidad» \\ Validation and reliability of the instrument «perception scale of physical education teacher's competencies in the assistance of disabled persons» \\ *Jorge Pérez-Contreras, *Cecilia Bahamonde-Pérez, **Carolina Pardo-Tamayo, ***Pablo Merino-Muñoz, ***Esteban Aedo-Muñoz \\ *Universidad Metropolitana de Ciencias de la Educación (Chile) **Universidad Santo Tomás (Chile)***Universidad de Santiago de Chile (Chile)
}

Resumen. El propósito del estudio fue determinar el grado de validez y fiabilidad del cuestionario Escala de Percepción de Competencia Docente en Educación Física en la Atención de las Personas en Situación de Discapacidad (ECoDEFyD). La muestra fue de tipo intencionada, compuesta por 37 profesores que imparten clases de Educación Física a nivel de educación básica y media. El instrumento original se modificó en base al juicio de expertos, y estos aprobaron su uso final alcanzando una validez de contenido aceptable. El instrumento modificado alcanzó una consistencia interna elevada $(\alpha>0.92)$ y una fiabilidad relativa excelente (coeficiente de correlación intraclase $=0.962)$, demostrando que el instrumento ECoDEFyD es un instrumento válido y fiable para medir la percepción de competencia y necesidades más urgentes frente a la inclusión de estudiantes con necesidades educativas especiales permanentes, de los profesores de Educación Física, resultando ser adecuado para tomar decisiones a partir de sus resultados.

Palabras claves: Estudio de validación, personas con discapacidad, profesor escolar, competencia profesional, percepción, necesidades educativas especiales.

\begin{abstract}
The purpose of this study was to determine the degree of validity and reliability of the questionnaire Scale of Perception of Teaching Competence in Physical Education in the Attention of People with Disabilities (ECoDEFyD). The sample was of intentional type, in 37 teachers who teach Physical Education classes at the basic and high school level. The original instrument was modified based on the judgment of experts, and they approved its final use, reaching an acceptable content validity. The modified instrument achieved high internal consistency $(\alpha>0.92)$ and excellent relative reliability (intra-class correlation coefficient $=0.962$ ), demonstrating that the ECoDEFyD instrument is a valid and reliable instrument to measure the perception of competence and the most urgent needs in the face of the inclusion of students with permanent special educational needs, of Physical Education teachers, proving to be adequate to make decisions based on their results. Keywords: Validation study, disabled persons, school teacher, professional competence, special educational needs.
\end{abstract}

\section{Introducción}

La normativa internacional de los últimos 20 años, ha sido clara respecto de garantizar la educación en igualdad de oportunidades a niños, niñas y adolescentes en situación de discapacidad (UNICEF, 2001). Estas normativas comprometen a los países a respetar el derecho de todos los niños a acceder a una educación integral y de calidad, sin ningún tipo de discriminación (UNICEF, 2001). La discapacidad es un término general que abarca las deficiencias, las limitaciones de la actividad y las restricciones de la participación (Ledezma,

Fecha recepción: 14-12-20. Fecha de aceptación: 16-09-21

Esteban Aedo Muñoz

esteban.aedo@usach.cl
2017). Por consiguiente, la discapacidad es un fenómeno complejo que refleja una interacción entre las características del organismo humano y las características en la sociedad en la que viven» (Cruz et al., 2010).

En Chile este propósito se ha desarrollado como una política pública, apoyado en la Ley19.284 de 1994, que abordaba la integración social de las personas con discapacidad, la cual fue reemplazada en el año 2010 por la Ley 20.422, que establece normas sobre igualdad de oportunidades e inclusión social de personas con discapacidad. Esta, en términos de educación, obliga al Estado a garantizar el acceso a las personas con discapacidad a los establecimientos públicos y privados del sistema de educación regular (Ley 20.422, Párrafo 2 , art. 34), los que deben contemplar planes para alumnos con Necesidades Educativas Especiales (NEE), y fomentar la participación de todos los docentes y demás 
integrantes de la comunidad educacional para lograr su aplicación. En este sentido, la inclusión de un estudiante con NEE asociado o no a una discapacidad, a un centro escolar regular implica que se generen modificaciones dentro y fuera de su aula dependiendo del tipo y grado de NEE que presente (Cruz et al., 2010).

En relación a lo anterior, y acorde al énfasis actual de abordar el currículo de forma inclusiva, el concepto de diversidad en la asignatura de educación física centra la mirada en las capacidades de cada persona y no en el déficit, por lo que el profesor de Educación Física (EFI) en su propuesta educativa debiera ser capaz de «abordar los diferentes componentes curriculares de forma flexible, contextualizada y pertinente a la composición de las y los estudiantes de cada curso, sus potencialidades y en relación con su contexto». Además de lograr «establecer estrategias pedagógicas diferenciadas que aseguren la accesibilidad de todos y todas a los objetivos de aprendizaje» (MINEDUC, 2015).

Sin embargo, gran parte de los profesores de EFI carecen de las competencias necesarias para guiar procesos de enseñanza aprendizaje en personas con NEE. Esto, debido a que durante su formación docente no han participado de actividades pedagógicas vinculadas a esta realidad.(Gangas \& Jiles, 2011; Duk et al., 2019; Hinrichsen, 2021).

El dominio de competencias docentes, que permitan la resolución de problemáticas teórico-practicas durante el desempeño profesional, deben ser adquiridas durante el pregrado. (Canadas et al., 2019). Las carreras de pedagogía, debe entregar aprendizajes que atiendan a los tiempos actuales, y de esta manera avanzar en una sociedad inclusiva, a partir de la practica en las unidades educativas y entregando una formación de excelencia, (Paez \& Hurtado Almonacid, 2019).

Es por esto que para hacer efectiva la inclusión, son necesarios cambios sustantivos en la formación inicial y continua de los profesores, que permitirá avanzar en una educación de calidad y que atienda a todos y cada uno de los educandos (Duk et al., 2019).

Bajo este argumento, sería necesario y pertinente, conocer si los profesores de EFI que imparten clases en el sistema nacional escolar, presentan o no las competencias para responder a los requerimientos de los y las estudiantes con NEE asociadas a discapacidad. Así también, descubrir cuáles son las necesidades que los docentes manifiestan para favorecer experiencias de aprendizaje significativo en sus estudiantes. Esto es posible mediante de la aplicación de un instrumento, válido y confiable que permita medir estas variables.
Actualmente en Chile no existen instrumentos validados que permitan evaluar el nivel de conocimientos de los profesores de educación física frente a la atención de estudiantes en situación de discapacidad y que consideren los nuevos planes y programas del Ministerio de Educación en relación al currículum nacional de la especialidad (MINEDUC, 2015).

La escasez de instrumentos que permitan evaluar la formación otorgada a los estudiantes de pedagogía en educacion física, y de esta manera determinar sus fortalezas y debilidades una vez egresados, impide poder realizar adecuaciones durante la formación (González Orb et al., 2019).

El primer problema que se presenta en los trabajos de investigación se centra en la construcción de instrumentos para la recolección de datos, ya que estos deberán recabar información válida y confiable. Válida, porque miden lo que se pretende medir y confiable porque pueden repetir la misma medida en condiciones similares, por lo que el valor del estudio dependerá de que esta información refleje fidedignamente el evento investigado (Corral, 2009).

Uno de instrumentos más robustos que cumplen con estos requisitos es el propuesto por Díaz del Cueto, (2009) que mide la percepción de competencia que manifiestan los profesores de EFI frente los alumnos con NEE (Díaz del Cueto, 2009), sin embargo este instrumento ha sido validado en otras realidades, y podría llevar a interpretaciones erróneas debido a que la traducción del cuestionario puede presentar diferencias culturales y de lenguaje (Ramada-Rodilla, Serra-Pujadas \& Delclós-Chanchet, 2013). Por tanto, el propósito de este estudio fue determinar el grado de validez y fiabilidad del cuestionario «Escala de Percepción de Competencia Docente en Educación Física en la Atención a las Personas Con Discapacidad» (ECoDEFyD) en profesores de Educación Física que se desempeñan en establecimientos de educación regular en la comuna de Macul de Santiago de Chile.

\section{Materiales y método}

Es esta investigación presenta un enfoque mixto secuencial de alcance exploratorio. El enfoque cualitativo se relacionó con el juicio de experto a través de un análisis observacional del instrumento, mientras que el enfoque cuantitativo se realizo mediante la aplicación de test-retest del instrumento adaptado del análisis observacional. La muestra estuvo compuesta por 37 profesores que imparten clases de educación física a ni- 
vel de educación básica y media de un total de 16 colegios de la comuna de Macul de Santiago de Chile. Se obtuvieron los consentimientos informados de los participantes, detallando objetivos, beneficios y riesgos de la investigación, además del resguardo de la información, confidencialidad y protección de identidad, siguiendo los principios éticos sobre investigaciones biomédica en humanos establecidos por la Declaración de Helsinki 2013 (World Medical Association Declaration of Helsinki: Ethical Principles for Medical Research Involving Human Subjects, 2013). El estudio obtuvo la aprobación por comité de ética institucional de la Universidad de Santiago de Chile N: 097/2020.

\section{Descripción de los instrumentos}

Instrumento Escala de Percepción de Competencia Docente en Educación Física en la Atención a las Personas Con Discapacidad (ECoDEFyD). Este cuestionario es una adaptación del utilizado previamente para la investigación: «El proceso de comunicación docente en Educación Física y su influencia sobre la adopción de hábitos de actividad físico-deportiva y de estilos de vida saludables entre la población escolar», realizada por el departamento de Educación Física, Deporte y Motricidad Humana de la Universidad Autónoma de Madrid (Díaz del Cueto, 2009). En la tabla $N^{\circ} 1$ se presentan las dimensiones que evalúa.

\section{Metodología de validación}

1. Validez de contenido por medio de juicio experto

Se seleccionó a tres expertos por conveniencia (Mcgartlanderg, Berg-Weger, Tebb, Lee \& Rauch, 2003), previo análisis de sus competencias, trayectoria docente y su relación con el tema en cuestión. Estos con basta experiencia en materias asociadas a la educación física, discapacidad e inclusión escolar y evaluación docente.

Se presentó el proyecto a cada uno de los expertos, con el propósito de explicar el contexto y los objetivos del estudio. Se les entregó la rúbrica de evaluación de juicio experto propuesta por Escobar-Pérez \& CuervoMartínez, (2008) junto con él instrumento original, para su evaluación (Escobar-Pérez \& Cuervo-Martínez, 2008).

2. Corrección y modificaciones del instrumento.

Una vez procesada la información, se llevaron a cabo las modificaciones en función de las puntuaciones y observaciones de los expertos, siguiendo las normativas y exigencias de las bases curriculares y de los planes y programas de Educación Física.
Realizadas las adecuaciones, estás fueron presentadas nuevamente a los expertos, la cual fue aprobada para su aplicación final.

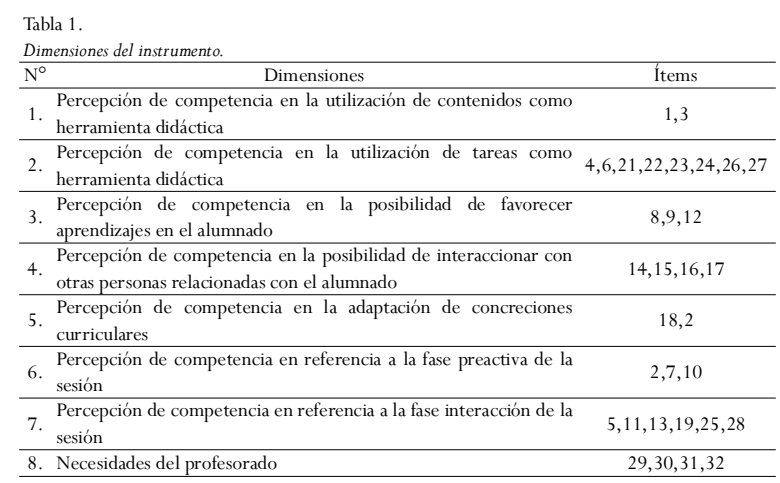

\section{Aplicación de los instrumentos}

La encuesta fue aplicada durante la última semana del mes Junio y segunda semana de Julio del 2020 por

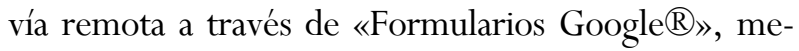
diante correo electrónico a cada uno de los profesores de EFI que impartan clases de forma regular en los colegios perteneciente a la Corporación Municipal de Educación de Macul, previa autorización del Director de Educación de la Comuna de Macul y de los directores de los establecimientos escolares definidos con anterioridad. Se aplicó dos momentos (test-retest), utilizando un intervalo de 2 semanas entre test y re-test (Serra-Mayoral \& Peña-Casanova, 2006). Se envió un correo electrónico a cada uno de los participantes, informando acerca de la investigación, donde a través de un enlace inserto en el correo, podían acceder al consentimiento informado. Una vez leído el consentimiento, los participantes tuvieron las opciones de aceptar o rechazar su participación de la investigación, porteriormente recibieron de forma automática un correo de respaldo y confirmación de su respuesta, para aquellos que aceptaron su participación se re-direccionó a una nueva pestaña con la encuesta. El formulario con la encuesta tuvo carácter anónimo, en 2 semanas se realizó el re-test a los mismos participantes, quienes aceptaron ser parte del estudio.

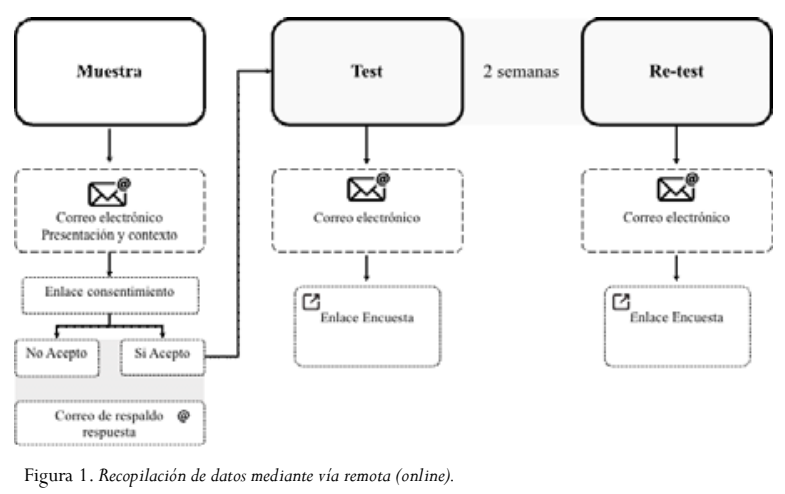




\section{Análisis Estadístico}

Para evaluar la consistencia del instrumento, se realizó una correlación alfa de $\operatorname{Cronbach}(\alpha)$ por dimensión y para el cuestionario total, utilizando valores; $\alpha>0.25$ indicaría baja confiabilidad; $\alpha>0.50$ la confiabilidad sería media o regular; $\alpha>0.70$ será aceptable y si $\alpha>0.90$ será elevada (Hernández, 2006). Se analizó la fiabilidad relativa del instrumento mediante el coeficiente de correlación intraclase (CCI) con sus respectivos intervalos de confianza (IC) al 95\%. La magnitud de los valores obtenidos en CCI se categorizarán a través de una escala cualitativa de tal forma que valores de fiabilidad de $<0.5$ se considerarán pobres, entre 0.5 a 0.74 moderados, 0.75 a 0.89 buenos y $>0.9$ excelentes (Koo \& Li, 2016). Todos los análisis estadísticos se realizaron a través del programa IBM SPSS statistics versión 25.0®.

\section{Resultados}

\section{Juicio de expertos}

Cada experto evaluó, cada uno de los ítems a través de una rúbrica de escala ordinal de 1 a 4 , siendo 4 la calificación más alta, y 1 la más baja. Los expertos valoraban los ítems acordes a 3 categorías: coherencia, relevancia y claridad. Por otro lado, valoraron las dimensiones de acuerdo con la categoría de «suficiencia». Estos valores se tomaron en consideración a la hora de realizar modificaciones de la encuesta original.

En relación con las observaciones de los expertos, se modificaron e incluyeron conceptos propios de las prácticas educativas vigentes como el Diseño Universal de Aprendizaje, las bases curriculares actuales y las modificaciones legales emanadas del MINEDUC como el decreto 67, todo esto basado en el juicio de los expertos. También se modificó la redacción de las preguntas, con el fin de mejorar el nivel de entendimiento de algunas categorías de la rúbrica de evaluación. El ítem 14 se dividió en dos y se eliminó el ítem 28 de la encuesta original.

\section{Modificaciones al instrumento}

En el primer apartado, «caracterización de la muestra» se realizaron cambios en los ítems 3, 4 y 5, donde solo se modificaron conceptos. El apartado de la encuesta «competencias docentes» constaba de 28 ítems, de los cuales los ítems 7, 9, 12, 14, 15, 16, 17, 18, 21, 26, 27 y 28 sufrieron modificaciones de redacción y conceptuales. El ítem 14 de la encuesta original fue dividido en dos ítems en la encuesta adaptada (ítems 14 y 15), y el 28 fue eliminado por no ser atingente a la dimensión que pretendía valorar. El tercer apartado de la encuesta llamado «Necesidades del profesorado» no sufrió modificaciones, por lo que quedó como la encuesta original (Anexo1).

\section{Fiabilidad del instrumento}

\section{a) Consistencia interna}

En la tabla 2, se pueden observar los resultados del análisis de fiabilidad (consistencia interna del primer test aplicado, donde las dimensiones 1, 2, 3, 7 y 8 tienen un nivel aceptable $(\alpha>0.7)$. Las dimensiones 4,5 y 6, obtuvieron un nivel regular $(\alpha=0.649, \alpha=0.679 \mathrm{y}$ $\alpha=0.677$ respectivamente). El instrumento, contando todos los ítems de este, obtuvo una consistencia elevada $(\alpha=0.920)$.

\begin{tabular}{|c|c|c|c|}
\hline \multicolumn{4}{|l|}{ Consistencia intern } \\
\hline Dimensión & $\mathrm{N}$ & $\alpha$ & Categorización \\
\hline D1 & 2 & 0.704 & Aceptable \\
\hline D2 & 8 & 0.835 & Aceptable \\
\hline D3 & 3 & 0.812 & Aceptable \\
\hline D4 & 4 & 0.649 & Regular \\
\hline D5 & 2 & 0.679 & Regular \\
\hline D6 & 3 & 0.677 & Regular \\
\hline D7 & 6 & 0.822 & Aceptable \\
\hline D8 & 4 & 0.719 & Aceptable \\
\hline Total & 32 & 0.920 & Elevada \\
\hline
\end{tabular}

b) Fiabilidad relativa

El ítem 1 y 31 obtuvieron fiabilidades pobres $(<0.5)$. Losítems 2, 3, 4, 5, 6, 8, 9, 12, 13, 17, 21, 23, 24, 25, 26, 29, 30 y 32 alcanzaron fiabilidades moderadas (entre

\begin{tabular}{|c|c|c|c|c|}
\hline \multirow[b]{2}{*}{ Ítem } & \multirow[b]{2}{*}{$\mathrm{CCI}^{*}$} & \multicolumn{2}{|c|}{$95 \%$ IC } & \multirow[b]{2}{*}{ Categorización } \\
\hline & & Limite Inferior & Limite Superior & \\
\hline 1 & 0.291 & -0.453 & 0.654 & Pobre \\
\hline 2 & 0.634 & 0.251 & 0.822 & Moderada \\
\hline 3 & 0.689 & 0.362 & 0.848 & Moderada \\
\hline 4 & 0.606 & 0.192 & 0.808 & Moderada \\
\hline 5 & 0.576 & 0.132 & 0.793 & Moderada \\
\hline 6 & 0.503 & -0.018 & 0.757 & Moderada \\
\hline 7 & 0.807 & 0.605 & 0.906 & Buena \\
\hline 8 & 0.647 & 0.277 & 0.828 & Moderada \\
\hline 9 & 0.662 & 0.307 & 0.835 & Moderada \\
\hline 10 & 0.863 & 0.719 & 0.933 & Buena \\
\hline 11 & 0.797 & 0.584 & 0.901 & Buena \\
\hline 12 & 0.705 & 0.395 & 0.856 & Moderada \\
\hline 13 & 0.704 & 0.394 & 0.856 & Moderada \\
\hline 14 & 0.911 & 0.818 & 0.957 & Excelente \\
\hline 15 & 0.787 & 0.563 & 0.896 & Buena \\
\hline 16 & 0.829 & 0.649 & 0.916 & Buena \\
\hline 17 & 0.683 & 0.35 & 0.845 & Moderada \\
\hline 18 & 0.773 & 0.534 & 0.889 & Buena \\
\hline 19 & 0.773 & 0.535 & 0.889 & Buena \\
\hline 20 & 0.76 & 0.508 & 0.883 & Buena \\
\hline 21 & 0.548 & 0.074 & 0.779 & Moderada \\
\hline 22 & 0.815 & 0.621 & 0.910 & Buena \\
\hline 23 & 0.611 & 0.204 & 0.810 & Moderada \\
\hline 24 & 0.582 & 0.144 & 0.796 & Moderada \\
\hline 25 & 0.623 & 0.228 & 0.816 & Moderada \\
\hline 26 & 0.696 & 0.377 & 0.851 & Moderada \\
\hline 27 & 0.819 & 0.629 & 0.911 & Buena \\
\hline 28 & 0.877 & 0.748 & 0.940 & Buena \\
\hline 29 & 0.614 & 0.209 & 0.811 & Moderada \\
\hline 30 & 0.743 & 0.474 & 0.875 & Moderada \\
\hline 31 & 0.470 & -0.086 & 0.741 & Pobre \\
\hline 32 & 0.730 & 0.447 & 0.868 & Moderada \\
\hline Total & 0.962 & 0.941 & 0.979 & Excelente \\
\hline
\end{tabular}


0.5 a 0.74), los ítems 7, 10, 11,15, 16, 18, 19, 20, 22, 27 y 28 fiabilidades buenas (entre 0.75 a 0.89 ), mientras que el ítem 14 obtuvo una fiabilidad excelente $(>0.9)$. Solo dos preguntas no alcanzaron un nivel de fiabilidad sobre 0.5 (moderada) que corresponden a un 6\% del total de ítems de la encuesta. La encuesta total tuvo una fiabilidad excelente (0.962). En tabla 3, se pueden observar los CCI con sus IC del 95\%.

\section{Discusión}

Llevar adelante un proceso de investigación científica, a través de la aplicación de instrumentos de medición, requiere que estos sean válidos y confiables, permitiendo que la información obtenida, tenga un mayor grado de objetividad (Flores-Ruiz et al., 2017; Villavicencio-Caparó et al.,2018)

Existen diversos estudios que abordan la formación, actitud y percepción de competencias docentes frente a la inclusión (Colmenero \& Pegalajar, 2015; Díaz del Cueto, 2009; Durán \& Sanz, 2015; Peña Anguita \& Peña Anguita, 2015), pero es necesario contar con un instrumento validado y confiable en el contexto nacional, debido a que el cuestionario modificado que valora la percepción de competencia que manifiestan los profesores de EFI frente los alumnos con NEE de Díaz del Cueto (2009) fue validado en otro contexto y realidad eduactiva.

Los principales hallazgos de este estudio fueron la validez de contenido otorgada por los expertos, los cuales luego de evaluar la encuesta, y sugerir modificaciones, reconocen su uso final (test-rest), como también el aceptable grado fiabilidad, mediante análisis de consistencia interna ( $\alpha$ de Cronbach) y fiabilidad relativa (CCI) elevadas (Almonacid-Fierro et al., 2018).

Debido a que la validez de contenido no puede ser cuantificada, sino más bien, es una cuestión de juicio y se estima de manera subjetiva o intersubjetiva (Corral, 2009) y en este caso los expertos aprobaron su uso final, se considera un instrumento válido a nivel de esta propiedad. En este sentido, Beaton, Bombardier, Guillemin \& Ferraz (2000), sugieren una metodología que primero traduce y adapta al idioma, cultura o contexto de interés, para luego ser evaluado y analizado por el juicio de expertos. A diferencia de esta metodología mencionada antes de cambiar o adaptar el instrumento, se dejó esta tarea a los expertos, debido a que los investigadores podrían no presentar las competencias requeridas para realizar esta adaptación.

En relación con la consistencia interna del instru- mento, el valor mínimo aceptable para el coeficiente alfa de Cronbach es 0.70 (Oviedo \& Campos-Arias, 2005). Usualmente se prefieren valores de alfa entre 0.80 y 0.90 (Streiner, 2003). Sin embargo, cuando no se cuenta con un mejor instrumento, se pueden permitir valores inferiores de alfa de Cronbach (Cortina, 1993). Lo anterior se evidencia en el estudio de GarcíaColl, Graupera-Sanz, Ruiz-Pérez \& Palomo-Nieto (2013) donde presentaron valores de « $\alpha »$ mayores a 0.7 , considerados como aceptables, con la excepción de una dimensión donde detallarón un valor de 0.63 , y en el total de ítems un valor elevado de 0.9 , lo que coincide con los valores presentados en el estudio de González Orb, et al (2019), donde se considera 0.7 como un valor respetable para el total de los ítems del test. Del mismo modo Fuentes, Errázuriz, Davison \& Cocio (2019), también consideraron como aceptables valores sobre 0.7 , obtenidos en casi todas las dimensiones, esto porque en una de ellas, se obtuvo un valor inferior de 0.69, mientras que Loewenthal \& Lewis (2018) detallan que, en dimensiones con menos de 10 ítems, es posible admitir valores sobre 0.6. En nuestro estudio 3 de 8 dimensiones no alcanzaron un nivel aceptable, llegando a un nivel regular $(0.7<\alpha>0.6)$, pero como no existe otro instrumento validado en español y américa latina que valore estas percepciones, adicionando que ninguna dimensión supera los 10 ítems, se podrían considerar aceptables. El instrumento total, es decir, la sumatoria de todas las dimensiones, detallaron una consistencia interna elevada, por lo que se considera adecuado para su aplicación general en Profesores de EFI e incluso para tomar decisiones diagnósticas (García-Coll et al., 2013).

Con respecto a la fiabilidad relativa, 30 de 32 ítems alcanzaron un nivel moderado a excelente, y los dos restantes un nivel pobre. Algunos autores han considerado resultados aceptables de CCI sobre 0.6 (MancholaGonzález, Bagur-Calafat \& Girabent-Farrés, 2017), otros sobre 0.7 (Arribas, 2004), pero estos valores dependerán de la escala de categorización cualitativa. La encuesta total obtuvo un nivel de fiabilidad excelente $(>0.9)$, por lo que tendría un nivel aceptable para ser replicado a través del tiempo (Arribas, 2004; Manterola Delgado, 2002).

Se reconoce como limitante del estudio, la aplicación del instrumento por vía remota, ya que esta metodología tiene como desventaja una menor tasa de respuesta en comparación con la aplicación de la encuesta de forma presencial (Díaz, 2012). También en relación al tamaño muestral para estudios de reproductibilidad, si bien nuestro estudio cumple con el mínimo estable- 
cido, que es mayor a 20 participantes (Hopkins, 2000), se aconseja un número mayor de 50 participantes de la población objetivo (Atkinson \& Nevill, 2001), por lo que una muestra mayor podría hacer que los ítems o dimensiones que obtuvieron bajos y pobres niveles de consistencia interna y fiabilidad relativa respectivamente podrían alcanzar un nivel mayor. Es recomendable realizar pruebas de validez de constructo como pruebas de convergencia-divergencia, análisis factorial exploratorio y confirmatorio, debido a que el análisis factorial confirmatorio requiere al menos 100 sujetos para dar resultados fiables en análisis factoriales de 2 factores y 500 en análisis de más factores (Villavicencio-Caparó et al., 2018), sin embargo en el presente estudio no fue posible acceder a esta cantidad de participantes.

\section{Conclusión}

A partir de los resultados obtenidos podemos concluir que el cuestionario ECoDEFyD es un instrumento válido y fiable, que permite conocer la percepción de competencia docente y las necesidades más urgentes de los profesores de educación física frente a la inclusión de estudiantes con NEE permanentes, asociadas a discapacidad. Debido a las adecuaciones realizadas, el protocolo de aplicación y el respaldo estadístico que el instrumento alcanzó, con una elevada consistencia interna y excelente estabilidad temporal, este resulta ser fiable a través de tiempo, como también mide las dimensiones que se pretende medir. Por lo tanto es posible que sea utilizado a nivel nacional y aplicado en profesores de la especialidad de educación física una vez egresados de la carrea y de esta manera conocer si durante su formación, han adquirido o no los conocimientos necesarios para el desarrollo de competencias que les permita responder a los requerimientos de los y las estudiantes con NEE asociadas a discapacidad. Vale destacar la importancia de poder mediante este instrumento elevar información que permita a las carreras de pedagogías en educación física, hacerse cargo de esta realidad, mediante una adecuación curricular que aborden esta problemática como parte del quehacer docente.

\section{Agradecimientos}

Agradecemos a la Universidad Metropolitana de Ciencias de la Educación y en especial al Departamento de Educación Física, Deportes y Recreación, representados en la Dra. Elisa Araya Cortéz por su incansable apoyo a la investigación educativa.

\section{Referencias}

Almonacid-Fierro,A., Feu,S., \& Carrizosa, M.V.(2018).Validation ofa questionnaire tomeasure didacticknowledge of contentin physical education teachers. Retos, 2041(34), 132-137.

Arribas, M. (2004). Diseñoy validación de cuestionarios. MatronasProf, 5(17), 23-29.https://ebevidencia.com/wp-content/uploads/ 2014/07/validacion_cuestionarios.pdf

Atkinson, G., \& Nevill,A. M.(2001). Selected issuesin the design and analysis of sport performance research. Journal of Sports Sciences, 19(10), 811-827.https://doi.org/10.1080/026404101317015447

Beaton, D. E., Bombardier, C., Guillemin, F., \& Ferraz, M. B. (2000). Guidelines for the process of cross-cultural adaptation of self-report measures. In Spine (Vol. 25, Issue 24, pp. 3186-3191). https: / / doi.org/10.1097/00007632-200012150-00014

Cañadas, L., Santos-Pastor, M.L., \& Castejón, F. J. (2018). Competencias docentes en la formación inicial del profesorado de educación física (Teaching competences in physical education teacher initial training). Retos, 2041(35), 284-288. https://doi.org/10.47197/ retos.v0i35.64812

Colmenero, M. J., \& Pegalajar, M. del C. (2015). Cuestionario para futuros docentes de educación secundaria acerca de las percepciones sobre atención a la diversidad: Construcción y validación del instrumento.EstudiossobreEducacion, 29, 165-189.https://doi.org/ 10.15581/004.29.165-189

Corral,Y.(2009).Validezy confiabilidad delosinstrumentos deinvestigación para la recolección de datos. Revista Ciencias dela Educación, 33, 228-247.http://servicio.bc.uc.edu.ve/educacion/revista/n33/ art12.pdf

Cortina, J. M. (1993). What is coefficient alpha? An examination of theory and applications. Journal of Applied Psychology, 78(1), 98-104. https://doi.org/10.1037//0021-9010.78.1.98

Cruz, A., Cruz, I., Vargas, M., Salazar, C., Manzo, E., \& Salazar, R. (2010). Laactuación del profesor de educación física en la integración educativa de alumnos con necesidades educativas especiales asociados a discapacidad motriz. Educación Física.Temas y Oposiciones., 1, 1-28. http:/ / www.argenmex.fahce.unlp.edu.ar/pdf/ EFArgenmex posicion 20 Cruz.pdf

Díazdel Cueto, M.(2009). Percepción de competencia del profesorado de educación física e inclusión. Revista Internacional de Medicina y Ciencias de laActividad Fisicay del Deporte, 9(1577-0354), 322-348. http: / / cdeporte.rediris.es / revista/revista35/ artpercepcion152.htm

Díaz,V.(2012).Ventajas e inconvenientes de la encuesta por internet. Papers: Revista de Sociología, 97(1), 193-223. https:/ / doi.org/ 10.5565/rev/papers/v97n1.71

Durán,D., \& Sanz,A.(2015). Dificultades del profesoradodeeducación física de educación secundaria ante el alumno con discapacidad. Revista Internacional de Medicina y Ciencias de la Actividad Física y del Deporte, 7(27). http:/ /hdl.handle.net/10486/3608

Duk,C., Cisternas,T., \& Ramos,L.(2019). Formacióndocentedesdeun enfoqueinclusivo.A 25 años dela declaración de salamanca, nuevos y viejos desafíos. Revista Latinoamericana de Educación Inclusiva, 13(2), 91-109.https://doi.org/10.4067/s0718-73782019000200091

Escobar-Pérez, J., \& Cuervo-Martínez,Á.(2008).Validezdecontenido 
y juicio de expertos: una aproximación a su utilización. Avances en Medición, 6(January 2008), 27-36. http:// www.humanas.unal.edu.co/psicometria/files/7113/8574/5708/ Articulo3_Juicio_de_expertos_27-36.pdf

Flores-Ruiz,E., Miranda-Novales,M.G., \&Villasís-Keever,M.Á.(2017). Theresearch protocolVI:How to choose the appropriate statistical test.Inferential statistics. RevistaAlergia Mexico, 64(3), 364-370.https:/ /doi.org/10.29262/ram.v64i3.304

Fuentes, L., Errázuriz, M. C., Davison, O.A., \& Cocio, A. (2019). Validación de una encuesta de actitudes de lectura en estudiantes de educación básica. Literatura y Lingüistica, 39, 225-250. https: / / doi.org/10.29344/0717621x.39.2012

Gangas,A., \& Jiles, D. (2011). Inclusión del estudiante con Discapacidad Visual en la clase de Educación Física [Universidad Austral de Chile]. http: / / cybertesis.uach.cl/tesis/uach/2011/ffg197i/doc/ ffg197i.pdf

García-Coll,V., Graupera-Sanz, J. L., Ruiz-Pérez, L. M., \& PalomoNieto, M. (2013). Inteligencia emocional en el deporte: validación española del Schutte Self Report Inventory (SSRI) en deportistas españoles. Cuadernos de Psicologia Del Deporte, 13(1), 25-36. https:/ /doi.org/10.4321/S1578-84232013000100004

González Orb, M., Molina Sotomayor, E., Ferreira Urzúa, M., Leiva Contardo, R., Martínez Salazar, C., Trujillo Galindo, H., Lobos González, M., Beltran Gonzalez,A.,VargasVitoria, R., Flandez Valderrama, J., \& Negron Molina, M. (2019). Construcción y validación de un instrumento para valorar la calidad de laformación de profesoresdeEducación Física en Chile. Retos, 2041(37), 312-319. https://doi.org/10.47197/retos.v37i37.69304

Hernández, E. (2006). Metodología de la Investigación: Cómo escribir una tesis.http://www.sld.cu/galerias/pdf/sitios/rehabilitacion-bio/ como_escribir_una_tesis.pdf

Hopkins, William G. (2000). Measures of reliability in sports medicine and science. Sports Medicine, 30(1), 1-15.https://doi.org/10.2165/ 00007256-200030010-00001

Koo,T.K., \& Li, M.Y.(2016).A Guideline of Selecting and Reporting Intraclass Correlation Coefficients for Reliability Research. Journal ofChiropractic Medicine, 15(2), 155-163.https://doi.org/10.1016/ j.jcm.2016.02.012

Loewenthal, K., \& Lewis, C.A.(2018).Anintroduction topsychological tests and scales. In An Introduction to Psychological Tests and Scales. https://doi.org/10.4324/9781315782980

Manchola-González,J., Bagur-Calafat, C.,\& Girabent-Farrés, M.(2017). Fiabilidad de la versión española del cuestionario de actividad física PAQ-C. Revista Internacional de Medicina y Ciencias de la Actividad Fisicay DelDeporte, 65(2017), 139-152.https://doi.org/10.15366/ rimcafd2017.65.010

Manterola Delgado, C. (2002). El proceso de medición con variables cualitativas y suaplicación en cirugía. Rev.Chil.Cir, 54(3), 307-315. https://www.cirujanosdechile.d/revista_anteriores/PDFCirujanos 2002_03/Cir.3_2002Variables Cualitativ.pdf

Mcgartland, D., Berg-weger, M., Tebb, S. S., Lee, E. S., \& Rauch, S. (2003). Objectifying ContentValidity: In SocialWork Research. SocialWorkResearch, 27(2), 94-104.https://doi.org/10.1093/swr/ 27.2.94

MINEDUC. (2015). Nuevas bases curriculares y programas de estudio $7^{\circ} y$ $8^{\circ}$ año de educación básica $/ 1^{\circ}$ y $2^{\circ}$ año de educación media; Cartilla de orientacionestécnicas (p. 17).https://bibliotecadigital.mineduc.d/ bitstream/handle/20.500.12365/14463/Cartilla-Curricular-FG1.pdf?sequence $=1$ \&isAllowed $=\mathrm{y}$

Lederma Ortega,D. (2017). Rehabilitaciónbasada en lacomunidad:una alternativa deinclusión y desarrollo comunitariopara personascon discapacidad. Boletín Informativo CEI, 4(1). http:// editorial.umariana.edu.co/revistas/index.php/ BoletinInformativoCEI/article/view/1272/1213

Oviedo,H., \& Campos-Arias,A.(2005).Aproximaciónal usodel coeficiente alfa de Cronbach. Revista Colombiana de Psiquiatría, 34(4), 572-580. http://www.scielo.org.co/ scielo.php?script $=$ sci_arttext\&pid $=$ S00 34 $74502005000400009 \& \operatorname{lng}=$ en\&tlng $=$ es.

Paez, J., \& HurtadoAlmonacid, J. (2018). Formacióninicial docenteen profesores de educación física. Levantamiento de competencias específicas a partir de las necesidades del medio educativo. Retos, 35(1), 61-66. https://doi.org/10.47197/ retos.v0i35.61887

PeñaAnguita, P., \& PeñaAnguita,D. (2015). Formación y actitud del profesoradode educación física con respectoa laatención a la diversidad del alumnado de primaria en la ciudad de jaén. EmásF: Revista Digital de Educación Física, 32, 20 -40.https: / / dialnet.unirioja.es/ servlet/articulo?codigo $=5381925$

MuñozHinrichsen,F.(2020). Discapacidadeinclusión, undesafíoparala educación física en Chile. Journal of Movement \& Health, 18(1). doi:http://dx.doi.org/10.5027/jmh-Vol18-Issue1(2021)art98

Ramada-Rodilla,J.M.,Serra-Pujadas, C., \&Deldós-Clanchet,G.(2013). Adaptación cultural y validación decuestionarios de salud: revisión y recomendaciones metodológicas. Salud Pública de México, 55(1), 57-66. http://www.scielo.org.mx/ scielo.php?script $=$ sci_arttext\&pid $=S 0036$ 36342013000100009\&lng $=$ es\&thg $=$ es.\%0A

Serra-Mayoral,A., \& Peña-Casanova, J. (2006). Fiabilidad test-reteste interevaluadordelTest Barcelona. Neurologia, 21(6), 277-281.http:/ /public-files.prbb.org/publicacions/02559081-11db-4227-b3c94b447c3f6527.pdf

Streiner, D. (2003). Starting at the Beginning: An introduction to coefficient alpha and internal consistency. Journal of Personality Assessment, 80(1), 99-103. https://doi.org/10.1207/ S15327752JPA8001_18

UNICEF. (2001). Ciclo de debates:Desafios de la política educacional «Inclusión de niños con discapacidad en la escuela regular» (M. Santelices \& L. M.Pérez(eds.)).https://www.unicef.cl/archivos_documento/ 47/debate8.pdf

Villavicencio-Caparó,E., Ruiz-García,V., \& Cabrera-Duffaut,A.(2018). Validación de cuestionarios. OdontologíaActiva Revista Cientifica, 1(3), 71.https://doi.org/10.26871/oactiva.v1i3.200

World Medical Association declaration of Helsinki: Ethical principlesfor medical research involving human subjects. (2013). Journal of the American MedicalAssociation, 310(20), 2191-2194.https://doi.org/ 10.1001/jama.2013.281053 